\title{
ON MIXED PHASES IN GAUGE THEORIES
}

\author{
V.L. CHERNYAK \\ Budker Institute for Nuclear Physics \\ 630090 Novosibirsk, Russia \\ E-mail: chernyak@inp.nsk.su
}

Talk given at "Continuous Advances in QCD-2002/Arkadyfest", honoring the 60-th birthday of ARKADY VAINSHTEIN;

17-23 May 2002, University of Minneapolis, Minnesota, USA

\begin{abstract}
In many gauge theories at different values of parameters entering Lagrangian, the vacuum is dominated by coherent condensates of different mutually non-local fields (for instance, by condensates of electric or magnetic charges, or by various dyons). It is argued that the transition between these "dual to each other" phases proceeds through the intermediate "mixed phase", having qualitatively different features. The examples considered include: ordinary YM, $N=1$ SYM, $N=1$ SQCD, and broken $N=2$ SYM and SQCD.
\end{abstract}




\section{To Arkady, my friend and teacher}

\section{1. $S U\left(N_{c}\right)-\mathrm{YM}$ at $\theta \neq 0$ and dyons}

The physics of this theory, and in particular the vacuum energy density $\bar{E}_{\text {vac }}\left(\theta, N_{c}\right)$, is supposed to be periodic in $\theta \rightarrow \theta+2 k \pi$. On the other hand, the standard large $N_{c}$-counting rules imply $\left(b_{o}=11 / 3\right)$ :

$$
\bar{E}_{v a c}=-N_{c}^{2} \frac{b_{o}}{4} \Lambda^{4} F\left(\theta / N_{c}\right),
$$

with $F(z \rightarrow 0)=1-c_{1} z^{2}+c_{2} z^{4}+\ldots{ }^{1} \quad$ It was first pointed out by E. Witten [1] (see also [2] for a similar behaviour in the "stringy-YM" theory) that the $N_{c}$-dependence in Eq.(1) and periodicity in $\theta \rightarrow \theta+2 \pi$ imply together that the function $F(z)$ should be nonanalytic in its argument. So, for instance, instead of Eq.(1), the more explicit form of dependence of $\bar{E}_{\text {vac }}$ on $\theta$ will rather look as:

$$
\bar{E}_{v a c}=-N_{c}^{2} \frac{b_{o}}{4} \Lambda^{4}\left\{\min \sum_{k} f\left(\frac{\theta+2 k \pi}{N_{c}}\right)\right\},
$$

with $f(z)$ being the "normal" analytic function.

The qualitative behaviour of the curve $\bar{E}_{v a c}\left(\theta, N_{c}\right)$ looks as follows. First, it is symmetric in $\theta \rightarrow-\theta$ and periodic in $\theta \rightarrow \theta+2 k \pi$. Further, it has minimum at $\theta=0$ and begins to increase with increasing $\theta>0$, as it follows from general considerations of the Euclidean functional integral determining this theory. It reaches its maximal value at $\theta=\pi$. The curve itself is continuous at this point, but there is a cusp so that $\bar{E}_{\text {vac }}$ begins to decrease in a symmetric way in the interval $\pi<\theta<2 \pi$, reaching the same minimal value at $\theta=2 \pi$.

As for the qualitative behaviour of the topological charge density, $\bar{P}_{v a c}\left(\theta, N_{c}\right)$, it follows from the relation: $\bar{P}_{v a c}\left(\theta, N_{c}\right) \sim d \bar{E}_{\text {vac }} / d \theta$, and looks as follows. First, it is antisymmetric in $\theta \rightarrow-\theta$ and periodic in $\theta \rightarrow \theta+2 k \pi$. So, it is zero at $\theta=0$ and increases with $\theta$ reaching its maximum value at $\theta=\pi$.

\footnotetext{
${ }^{1}$ The numerical coefficient $c_{1}$ is positive, but it is a dynamical quantity and can not be determined from general considerations alone.
} 
There is a discontinuity at this point, so that the curve jumps to the same but negative value as $\theta$ overshoots $\pi$, and increasing in a symmetric way reaches zero at $\theta=2 \pi$.

The above described nonanalytic (cusped) behaviour of $\bar{E}_{v a c}\left(\theta, N_{c}\right)$ along the real $\theta$-axis agrees, in particular, with the asymptotic behaviour of $\bar{E}_{v a c}\left(\theta, N_{c}\right)$ at large imaginary values of $\theta, i \theta / N_{c}=\tilde{\theta} / N_{c} \gg 1$, obtained in [3]:

$$
\bar{E}_{v a c}\left(\theta, N_{c}\right) \sim-N_{c}^{2} \Lambda^{4} \exp \left\{\frac{4}{b_{o}} \frac{\tilde{\theta}}{N_{c}}\right\} .
$$

It is seen from Eq.(3) that $\bar{E}_{v a c}\left(\theta, N_{c}\right)$ is not naturally periodic at $\tilde{\theta} \rightarrow$ $\tilde{\theta} \pm 2 i \pi$. Rather, it implies that periodic $\bar{E}_{v a c}\left(\theta, N_{c}\right)$ is analytic in the strip $-\pi<\operatorname{Re} \theta<\pi$ in the complex $\theta$-plane, and is glued then periodically strip by strip.

The natural physical interpretation explaining the origin of the above described cusped behaviour of $\bar{E}_{v a c}(\theta)$ along the real $\theta$-axis has been proposed in [3], and looks as follows.

Let us suppose the "standard" picture of the confinement mechanism to be valid, i.e. those of the dual superconductor. By this we imply here the dynamical mechanism with composite (naturally adjoint) Higgs field which determines the formation of $U(1)^{N_{c}-1}$ from the original $S U\left(N_{c}\right), S U\left(N_{c}\right) \rightarrow$ $U(1)^{N_{c}-1}$, and besides the $U_{i}(1)$-magnetically charged excitations (monopoles) condense. We will be interested to trace the qualitative behaviour of this vacuum state in its dependence of $\theta$. For this, it will be sufficient to consider the "first" U(1)-charge only with its monopoles, the dual photon and correspond$\operatorname{ing} g^{ \pm}$gluons as if it were the $\mathrm{SU}(2)$ theory, because $\theta$ is $S U\left(N_{c}\right)$-singlet and all other $U(1)$ charges will naturally behave the same way under variation of $\theta$.

As has been shown by E. Witten [4], the pure monopole $\mathrm{M}=$ (magnetic charge $=1$, electric charge $=0$ ) at $\theta=0$ turns into the dyon with charges $d_{1}^{\theta}=(1, \theta / 2 \pi)$ at $\theta \neq 0$. So, the coherent condensate of monopoles in the vacuum at $\theta=0$ turns into the condensate of $d_{1}^{\theta}$-dyons as $\theta$ starts to deviate from zero, and the vacuum energy density begins to increase for this reason.

It is a specific property of our system that there are two types of condensates made of the dyons and antidyons with the charges: $\{(1,1 / 2) ;(-1,-1 / 2)\}$ and $\{(1,-1 / 2) ;(-1,1 / 2)\}$, and having the same energy density. This can be seen, for instance, as follows. Let us start from the pure monopole condensate at $\theta=0$ and let us move anticlockwise along the path: $\theta=0 \rightarrow \theta=\pi$. 
The vacuum state will consist of $\left(1, \frac{1}{2}\right)$ - dyons and $\left(-1,-\frac{1}{2}\right)$ - antidyons. Let us move now clockwise along the path: $\theta=0 \rightarrow \theta=-\pi$. The vacuum state will consist now of $\left(1,-\frac{1}{2}\right)$ - dyons and $\left(-1, \frac{1}{2}\right)$ - antidyons. Because the vacuum energy density is even under $\theta \rightarrow-\theta$, these two vacuum states are degenerate. ${ }^{2}$

Besides, these two states belong to the same world as they are reachable one from another through a barrier, because there are electrically charged gluons, $g^{ \pm}=(0, \pm 1)$, which can recharge these $(1, \pm 1 / 2)$ - dyons into each other. In contrast, the two vacuum states, $|\theta\rangle$ and $|-\theta\rangle$ at $\theta \neq 0, \pi$ are unreachable one from another and belong to different worlds, as there is no particles in the spectrum capable to recharge the $(1, \pm \theta / 2 \pi)$ - dyons into each other.

Thus, the vacuum state becomes twice degenerate at $\theta=\pi$, so that the "level crossing" (in the form of rechargement: $\left\{d_{1}=(1,1 / 2), \bar{d}_{1}=\right.$ $\left.(-1,-1 / 2)\} \rightarrow\left\{d_{2}=(1,-1 / 2), \bar{d}_{2}=(-1,1 / 2)\right\}\right)$ can take place if this will lower the energy density at $\theta>\pi$. And indeed it lowers, and this leads to a casp in $\bar{E}_{\text {vac }}(\theta)$. At $\theta>\pi$ the vacuum is filled now with the coherent condensate of new dyons with the charges: $d_{2}^{\theta}=(1,-1+\theta / 2 \pi), \bar{d}_{2}^{\theta}=(-1,1-\theta / 2 \pi)$. As $\theta$ increases further, the electric charge of these $d_{2}^{\theta}$-dyons decreases, and the vacuum energy density decreases with it. Finally, at $\theta=2 \pi$ the $d_{2}^{\theta}$-dyons (which were the $(1,-1)$-dyons at $\theta=0$ ) become pure monopoles, and the vacuum state becomes exactly as it was at $\theta=0$, i.e. the same condensate of pure monopoles and antimonopoles.

We emphasize that, as it follows from the above picture, it is wrong to imagine the vacuum state at $\theta=2 \pi$ as, for instance, a condensate of dyons with the charges $(1,-1)$, degenerate in energy with the pure monopole condensate at $\theta=0 .{ }^{3}$

\footnotetext{
${ }^{2}$ The existence of two vacuum states at $\theta=\pi$ does not follow from the symmetry considerations alone, like $\bar{E}_{v a c}(\theta)=\bar{E}_{v a c}(-\theta)$ and $\bar{E}_{v a c}(\theta)=\bar{E}_{v a c}(\theta+2 \pi k)$. It is sufficient to give a counterexample. So, let us consider the SU(2) Yang-Mills together with the Higgs doublet with large vacuum condensate. In this case the $\theta$-dependence of the vacuum energy density is due to a rare quasiclassical gas of instantons, and is $\sim \cos (\theta)$. All the above symmetry properties are fulfilled, but there is only one vacuum state at $\theta=\pi$. (See also the end of this section.)

${ }^{3}$ In this respect, the widely used terminology naming the two singularity points $u=$ $\pm \Lambda^{2}$ on the $N=2 S U\left(N_{c}=2\right)$ SYM moduli space as those where monopoles and respectively dyons become massless, is not quite adequate (and may be dangerous for this reason, leading to wrong conclusions). It is based on quantum numbers $\vec{n}=\left(n_{m}, n_{e}\right)$ of corresponding fields, and these quantum numbers are always the same independently of
} 
Physically, the above rechargement process will appear as a typical first order phase transition. After $\theta$ overshoots $\pi$, in a space with the coherent condensate of $d_{1}=(1,1 / 2)$ - dyons and $\bar{d}_{1}=(-1,-1 / 2)$ - antidyons, the bubbles will appear with the coherent condensate of $d_{2}=(1,-1 / 2)$ - dyons and $\bar{d}_{2}=(-1,1 / 2)$ - antidyons deep inside each bubble, and with a transition region surface (domain wall) through which the averaged densities of two type dyons interpolate smoothly. These bubbles expand then over all the space through the rechargement process $d_{1}+\bar{d}_{1} \rightarrow d_{2}+\bar{d}_{2}$ occuring on a surface of each bubble. This rechargement can be thought as going through a copious "production" of charged gluon pairs $g^{+} g^{-}$, so that the underlying processes are: $\left[d_{1}=(1,1 / 2)\right]+\left[g^{-}=(0,-1)\right] \rightarrow\left[d_{2}=(1,-1 / 2)\right]$ and $\left[\bar{d}_{1}=(-1,-1 / 2)\right]+\left[g^{+}=(0,1)\right] \rightarrow\left[\bar{d}_{2}=(-1,1 / 2)\right]$.

Some analogy with the simplest Schwinger model may be useful at this point, in connection with the above described rechargement process. Let us consider first the pure $Q E D_{2}$ without finite mass charged particles, and let us put two infinitely heavy "quarks" with the charges $\pm \theta / 2 \pi$ (in units of some $e_{o}$ ) at the edges of our space. It is well known [5] that this is equivalent to introducing the $\theta$-angle into the $Q E D_{2}$ Lagrangian. As a result, there is the empty vacuum at $\theta=0$, and the long range Coulomb "string" at $\theta \neq 0$. The vacuum energy density behaves as: $\bar{E}_{v a c}(\theta)=C_{o} e_{o}^{2} \theta^{2}, C_{o}=$ const, at any $0 \leq \theta<\infty$.

Let us add now some finite mass, $m \gg e_{o}$, and of unit charge $e_{o}$ field $\phi$ to the Lagrangian. When there are no external charges, this massive charged

the point of the moduli space we are staying in, and are not direct physical observables. In contrast, the standard physical terminology is based on charges $\vec{g}=\left(g_{m}, g_{e}\right)$ which are the direct physical observables because, by definition, the Coulomb interaction of two particles is proportional to product of their charges, not quantum numbers. In distinction from quantum numbers, the values of charges depend on the point of the moduli space, due to Witten's effect.

To illustrate, let us start from the vacuum $u=\Lambda^{2}$ where, by definition, the massless particles are pure monopoles and let us move, for instance, along a circle to the point $u=-\Lambda^{2}$. On the way, the former massless monopole increases its mass because it becomes the $d_{1}^{\theta}=(1, \theta(u) / 2 \pi)$ - dyon (here $\theta(u) / 2 \pi=\operatorname{Re} \tau(u)$ ). At the same time, the former massive $d_{2}^{o}=(1,-1)$ - dyon diminishes its mass as it becomes the $d_{2}^{\theta}=(1,-1+\theta(u) / 2 \pi)$ dyon. When we reach the point $u=-\Lambda^{2}$, i.e. $\theta(u)=2 \pi$, the former dyon becomes massless just because it becomes the pure monopole here. So, an observer living in the world with $u=-\Lambda^{2}$ will also see the massless monopoles (not dyons, and this is distinguishable by their Coulomb interactions between themselves and with other dyons), exactly as those living in the world with $u=\Lambda^{2}$. 
field can be integrated out, resulting in a small charge renormalization. But when the above quarks are introduced, the behaviour of $\bar{E}_{\text {vac }}(\theta)$ becomes nontrivial. The charge of the external quark tends to $1 / 2$ as $\theta$ approaches $\pi$. As $\theta$ overshoots $\pi$ it becomes preferable to produce a pair of $\phi$ - particles, $\phi^{+} \phi^{-}$, from the vacuum. They separate so that to recharge the external quarks: $( \pm 1 / 2) \rightarrow(\mp 1 / 2)$ (without changing the volume energy), and the external charges become equal $(\theta / 2 \pi-1)$ and $(-\theta / 2 \pi+1)$ at $\theta>\pi$. As a result of this rechargement, there appears a cusp in $\bar{E}_{v a c}(\theta)$ and it begins to decrease at $\theta>\pi$, so that the former "empty" vacuum is reached at $\theta=2 \pi$. Therefore, the behaviour of $\bar{E}_{v a c}(\theta)$ will be: $\bar{E}_{v a c}(\theta)=C_{o} e_{o}^{2} \cdot\left\{\min _{k}(\theta+2 \pi k)^{2}\right\}$, so that $\bar{E}_{\text {vac }}(\theta)=C_{o} e_{o}^{2} \theta^{2}$ at $0 \leq \theta \leq \pi$, and $\left.\bar{E}_{\text {vac }}(\theta)=C_{o} e_{o}^{2}(2 \pi-\theta)^{2}\right)$ at $\pi \leq \theta \leq 2 \pi$.

Let us return however to our dyons. The above described picture predicts also a definite qualitative behaviour of the topological charge density, $\bar{P}(\theta)$. At $0<\theta<\pi$, i.e. in the condensate of the $d_{1}^{\theta}=(1, \theta / 2 \pi)$ - dyons and $\bar{d}_{1}^{\theta}=(-1,-\theta / 2 \pi)-$ antidyons, the product of signs of the magnetic and electric charges is positive for both $d_{1}^{\theta}$ - dyons and $\bar{d}_{1}^{\theta}$ - antidyons. Thus, these charges give rise to the correlated field strengths: $\vec{E} \| \theta \vec{H}, \vec{E} \cdot \vec{H}>0$, and both species contribute a positive amount to the mean value of the topological charge density, so that $\bar{P}_{1}(\theta)>0$ and grows monotonically with $\theta$ in this interval following increasing electric charge $\sim \theta / 2 \pi$ of the dyon.

On the other side, at $\pi<\theta<2 \pi$, i.e. in the condensate of the $d_{2}^{\theta}=$ $(1,-1+\theta / 2 \pi)$ - dyons and $\bar{d}_{2}^{\theta}=(-1,1-\theta / 2 \pi)$ - antidyons, the product of signs of the magnetic and electric charges is negative for both $d_{2}^{\theta}$ - dyons and $\bar{d}_{2}^{\theta}$ - antidyons. Thus, both species contribute a negative amount to $\bar{P}_{2}(\theta)$, such that: $\bar{P}_{2}(\theta)=-\bar{P}_{1}(2 \pi-\theta)$, and $\bar{P}(\theta)$ jumps reversing its sign at $\theta=\pi$ due to rechargement.

On the whole, it is seen that the cusped behaviour of $\bar{E}_{\text {vac }}(\theta)$ and discontinuous behaviour of $\bar{P}(\theta)$ appear naturally in this picture of the confinement mechanism in $S U\left(N_{c}\right)$ - YM theory, and are exactly the same that are expected from simplest general considerations and were described in the beginning of this section.

Clearly, at $0 \leq \theta<\pi$ the condensate made of only the $d_{1}^{\theta}=(1, \theta / 2 \pi)$ - dyons (recalling also for a possible charged gluon pair production) can screen the same type $d_{k}^{\theta}=[$ const $(1, \theta / 2 \pi)+(0, k)]$ - test dyon only $(k=$ $0, \pm 1, \pm 2, \ldots ;$ and the same for the $d_{2}^{\theta}$ - dyons at $\left.\pi<\theta \leq 2 \pi\right)$. So, the 
heavy quark-antiquark pair will be confined at $\theta \neq \pi$.

New nontrivial phenomena arise at $\theta=\pi$. Because there are two degenerate states, i.e. the condensates of $\left(1, \pm \frac{1}{2}\right)$ - dyons (and antidyons), a "mixed state" configuration becomes possible with, for instance, each condensate filling a half of space only, and with the domain wall interpolating between them. The simplest reasonings about the energy scales involved in this domain wall are as follows. The masses of relevant gluons $g^{ \pm}=(0, \pm 1)$ and both dyons $(1, \pm 1 / 2)$ coexisting together in the core of the domain wall are naturally $\simeq \Lambda_{Y M}$, and so of the same size will be increase in energy density. Besides, there are $\left(N_{c}-1\right)$ independent $U_{i}(1)$ charges. On the whole, therefore, the domain wall tension will be $T \sim N_{c} \Lambda_{Y M}^{3}$, while its typical width will be $\sim 1 / \Lambda_{Y M}$.

Physically, the above domain wall represents "a smeared rechargement", i.e. smeared over space interpolation of electrically charged degrees of freedom between their corresponding vacuum values, resulting in a smooth variation of the averaged densities of both type dyons $(1, \pm 1 / 2)$ through the domain wall. Surprisingly, there is no confinement inside the core of such domain wall.

The reason is as follows. Let us take the domain wall interpolating along the z-axis, so that at $z \rightarrow-\infty$ there is the main coherent density of $d_{1}=$ $(1,1 / 2)$ - dyons, and at $z \rightarrow \infty$, - that of $d_{2}=(1,-1 / 2)$ - dyons. As we move from the far left to the right, the density of $d_{1}$-dyons decreases and there is also a smaller but increasing incoherent density of $d_{2}$-dyons. This small amount of $d_{2}$-dyons is "harmless", in the sense that its presence does not result in the screening of the corresponding charge. The reason is clear: the large coherent density of $d_{1}$-dyons confines the $d_{2}$-dyons so that they can not move freely and appear only in the form of rare and tightly connected neutral pairs $\bar{d}_{2} d_{2}$, with different pairs fluctuating independently of each other. As we are going further to the right, the density of these neutral pairs grows and their typical size increases (although they are still confined), because the main density of $d_{1}$-dyons decreases. Finally, at some distance from the centre of the wall the percolation takes place, i.e. the $d_{2}$-dyons form a continuous coherent network and become released, so that the individual $d_{2}$-dyon can travel freely to arbitrary large distances (in the transverse xy-plane) but only within its network. And in this percolated region the coherent network of $d_{1}$-dyons still survives, so that these two coherent networks coexist in space and form the new "mixed phase" with qualitatively different properties. 
This is a general feature, and each time when there will coexist coherent condensates of two mutually non-local fields, they will try to confine each other, and will resemble the above described case.

The above mixed phase shares some features in common with the mixed state of the type-II superconductor in the external magnetic field. The crucial difference is that the magnetic flux is sourceless inside the superconductor, while in the above described mixed phase there are real dual to each other charges, each type living within its network.

As we move further to the right, the density of $d_{2}$-dyons continue to increase while those of $d_{1}$ continue to decrease. Finally, at the symmetric distance to the right of the wall centre the "inverse percolation" takes place, i.e. the coherent network of $d_{1}$-dyons decays into separate independently fluctuating neutral droplets whose average density (and size) continue to decrease with increasing $z$. Clearly, the picture on the right side repeats in a symmetric way those on the left one, with the $d_{1}$ and $d_{2}$ dyons interchanging their roles. ${ }^{4}$

Let us consider now the heavy test quark put inside the core of the domain wall, i.e. inside the mixed phase region. Clearly, this region has the properties of the "double Higgs phase". Indeed, because the (two-dimensional) charges of two dyons, $(1,1 / 2)$ and $(1,-1 / 2)$, are linearly independent, polarizing itself appropriately this system of charges will screen any external charge put inside, and the quark one in particular.

Finally, if the test quark is put far from the core of the wall, the string will originate from this point making its way toward the wall, and will be screened inside the mixed phase (i.e. the double Higgs) region. It should be emphasized however that, as it is clear from the above explanations, if this test quark is moved further inside the core of the another wall, then its flux will be screened and nothing will support this string. So, the electric string can not be stretched between two such domain walls.

Let us point out finally that the assumption about the confinement prop-

\footnotetext{
${ }^{4}$ Evidently, if we replace the above domain wall with fixed $\theta=\pi$ by the domain wall of the light axion field $a(z), m_{a} \ll \Lambda_{Y M}$, interpolating between $a=0$ at $z \rightarrow-\infty$ and $a=2 \pi$ at $z \rightarrow \infty$ with $a(z=0)=\pi$, all the properties will remain the same in the core, i.e. at $|z| \lesssim($ several $) \Lambda_{Y M}^{-1}$. The main difference will be that the condensates of $d_{1}=(1,1 / 2)$ and $d_{2}=(1,-1 / 2)$ - dyons will turn into condensates of pure monopoles at corresponding sides of large distances $|z| \gg 1 / m_{a}$ (see also the next section).
} 
erty of the $S U\left(N_{c}\right)$ YM theory is not a pure guess, as the above discussed nonanalytic (i.e. cusped) behaviour of the vacuum energy density, $\bar{E}_{v a c}\left(\theta, N_{c}\right)$, is a clear evidence for a phase transition at some finite temperature. Indeed, at high temperatures the $\theta$ - dependence of the free energy density in the gluon plasma is under control and is: $\sim T^{4}(\Lambda / T)^{N_{c} b_{o}} \cos \theta$, due to rare gas of instantons. It is important for us here that it is perfectly analytic in $\theta$, and that the form of its $\theta$ dependence is T-independent, i.e. it remains to be $\sim \cos \theta$ when the temperature decreases. On the opposite side at $T=0$, i.e. in the confinement phase, the $\theta$ - dependence is nonanalytic and, clearly, this nonanalyticity survives at small temperatures as there are no massless particles in the spectrum. So, there should be a phase transition (confinement - deconfinement) at some critical temperature, $T_{c} \simeq \Lambda$, where the $\theta$ dependence changes qualitatively.

\section{2. $\quad N=1 \quad S U\left(N_{c}\right) \quad S Y M$}

In this theory the residual non-anomalous discrete axial symmetry is broken spontaneously, so that there are $N_{c}$ vacuum states differing by the phase of the gluino condensate $[6],[7]$ :

$$
\langle 0|\lambda \lambda| 0\rangle_{k} \sim N_{c} \Lambda^{3} \exp \left\{i \frac{2 \pi k}{N_{c}}\right\} .
$$

Besides, it is widely believed that this theory is confining, similarly to the usual YM- theory. In what follows, we will suppose that the confinement mechanism here is the same as in the previous section, i.e. those of the dual superconductor. Our purpose in this section will be to describe qualitatively the physical properties of domain walls interpolating between the above vacua and, in particular, their ability to screen the quark charge [8].

For this, let us consider the effective theory obtained by integrating out all degrees of freedom except for the composite chiral field $S=\left(W_{\alpha}^{2} / 32 \pi^{2} N_{c}\right), S=$ $(\lambda \lambda, \cdots) / 32 \pi^{2} N_{c}=(\rho \exp \{i \phi\}, \ldots)$ (i.e. the integration proceeds with the constraint that the field $S$ is fixed, [8]). In this set up, the form of the superpotential in the Lagrangian for the field $S$ can then be simply obtained and coincides with those of Veneziano-Yankielowicz [6]: $W \sim S \ln \left(S^{N} / \Lambda^{3 N}\right)$, resulting in the gluino condensation, see Eq.(4).

Because the field $N_{c} \phi$ in SYM is the exact analog of $\theta$ in the ordinary YM, the physical interpretation and qualitative behaviour of $\bar{E}_{v a c}(\theta)$ in the 
YM- theory described in the previous section can be transfered now to SYM, with only some evident changes:

a) $\bar{E}_{\text {vac }}(\theta) \rightarrow U\left(N_{c} \phi\right)$, and it is not the vacuum energy density now but rather the potential of the field $\phi$;

b) if we start with the condensate of pure monopoles at $\phi=0$, the rechargement $d_{1}^{\phi}=\left(1, N_{c} \phi / 2 \pi\right) \rightarrow d_{2}^{\phi}=\left(1,-1+N_{c} \phi / 2 \pi\right)$ and the cusp in $U\left(N_{c} \phi\right)$ will occur now at $\phi=\pi / N_{c}$, so that at $\phi=2 \pi / N_{c}$ we will arrive at the next vacuum with the same pure monopole condensate but with shifted phase of the gluino condensate.

Let us consider now the domain wall interpolating along $z$-axis between two nearest vacua with $k=0$ and $k=1$, so that $\phi(z) \rightarrow 0$ at $z \rightarrow-\infty$, and $\phi(z) \rightarrow 2 \pi / N_{c}$ at $z \rightarrow \infty$. There is a crucial difference between this case and those described just above where the field $\phi$ was considered as being space-time independent, i.e. $\phi(z)=$ const. The matter is that the system can not behave now in a way described above (which allowed it to have a lowest energy $U\left(N_{c} \phi\right)$ at each given value of $\phi(z)=\phi=$ const): i.e. to be the pure coherent condensate of $d_{1}^{\phi}$ - dyons at $0 \leq \phi<\pi / N_{c}$, the pure coherent condensate of $d_{2}^{\phi}$ - dyons at $\pi / N_{c}<\phi \leq 2 \pi / N_{c}$, and to recharge suddenly at $\phi=\pi / N_{c}$. The reason is that the fields corresponding to electrically charged degrees of freedom also become functions of $z$ at $q=\int d z\left[d \phi_{d w}(z) / d z\right] \neq 0$. So, they can not change abruptly now at $z=0$ where $\phi_{d w}(z)$ goes through $\pi / N_{c}$, because their kinetic energy will become infinitely large in this case. Thus, the transition will be smeared necessarily.

The qualitative properties of the domain wall under consideration here will be similar to those described in the previous section. The main difference is that $\theta$ was fixed at $\pi$ in sect.1, while $N_{c} \phi_{d w}(z)$ acts like the axion field, i.e. it varies here between its limiting values, and the electric charges of dyons follow it.

So, at far left there will be a large coherent condensate of $d_{1}^{\phi}=\left(1, N_{c} \phi / 2 \pi\right)$ dyons (pure monopoles at $z \rightarrow-\infty$ ), and a small incoherent density of $d_{2}^{\phi}=\left(1,-1+N_{c} \phi / 2 \pi\right)$-dyons. ${ }^{5}$ The $d_{2}^{\phi}$-dyons can not move freely in this region as they are confined, and appear as a rare and tightly connected neutral pairs $\bar{d}_{2}^{\phi} d_{2}^{\phi}$ only. Therefore, their presence does not result in the screening of the corresponding charge. As we move to the right, the density of $d_{1}^{\phi}$-dyons decreases while those of $d_{2}^{\phi}$ - increases. These last move more

\footnotetext{
${ }^{5}$ Other possible dyons play no role in the transition we consider, and we will ignore them.
} 
and more freely, but are still confined. Finally, their density reaches a critical value at $z=-z_{o}$, so that a percolation takes place and the $d_{2}^{\phi}$-dyons form a continuous coherent network within which the individual $d_{2}^{\phi}$-dyons can move freely to any distance (in the transverse xy-plane). At the same time, there still survives a coherent condensate of $d_{1}^{\phi}$-dyons, which still can freely move individually within their own network.

At the symmetrical point $z=z_{o}$ to the right of the domain wall centre at $z=0$, the "inverse percolation" takes place, so that the coherent connected network of $d_{1}^{\phi}$-dyons decays into separate independently fluctuating neutral droplets, whose density (and size) decreases with further increasing z. At large $\mathrm{z}$ we arrive at the vacuum state with a large coherent condensate of monopoles (former $(1,-1)$-dyons at large negative $\mathrm{z}$ ).

Now, let us consider what happens when a heavy quark is put inside the core of the domain wall. The crucial point is that there is a mixture of all four dyon and antidyon species (of all $N_{c}-1$ types): $d_{1}^{\phi}=\left(1, N_{c} \phi / 2 \pi\right), \bar{d}_{1}^{\phi}=$ $\left(-1,-N_{c} \phi / 2 \pi\right), d_{2}^{\phi}=\left(1,-1+N_{c} \phi / 2 \pi\right)$ and $\bar{d}_{2}^{\phi}=\left(-1,1-N_{c} \phi / 2 \pi\right)$ in this percolated region, with each dyon moving freely inside its coherent network. So, this region has the properties of "the double Higgs phase", as here both the $d_{1}^{\phi}$ and $d_{2}^{\phi}$-dyons are capable to screen corresponding charges. And because the charges of $d_{1}^{\phi}$ and $d_{2}^{\phi}$-dyons are linearly independent, polarizing itself appropriately this mixture of dyons will screen any test charge put inside, the heavy quark one in particular.

If the test quark is put at far left (or right) of the wall, the string will originate from this point making its way towards a wall, and will disappear inside the core of the wall, i.e. in the mixed phase region where the string flux will be screened. And similarly to the previous section, the string can not be stretched between two domain walls.

The above described explanation of the physical phenomena resulting in quark string ending in the wall differs from both, those described by E. Witten in [9] and those proposed by I. Kogan, A. Kovner and M. Shifman in [10] (see also the footnote 3 ).

\section{3. $\quad N=1 \quad S U(2), N_{F}=1 \quad \mathrm{SQCD}$}

As previously, we will imply here that there is confinement of electric charges in the $N=1$ pure SYM- theory (see previous sections). Then, there will be three phases in this $N=1$ SQCD - theory, depending on the value of $m$ - the mass parameter of the quark [8]. 
At small $m \ll \Lambda$, there will be the usual electric Higgs phase, with the large quark condensate $\langle\bar{Q} Q\rangle \sim\left(\Lambda^{5} / m\right)^{1 / 2}$, and light quark composite fields $(\bar{Q} Q)^{1 / 2}$ with masses $\sim m$. The effective low energy Lagrangian for these fields is those of Affleck-Dine-Seiberg [11].

The heavy magnetically charged excitations (monopoles) will be confined, and so the monopoles will appear as rare and tightly bound neutral pairs only, with different pairs fluctuating independently of each other. ${ }^{6}$

With increasing $m$ the quark condensate and the monopole mass decrease, while the density of monopole pairs and their typical size increase. At some value $m=c_{1} \Lambda$ the percolation of the monopole droplets takes place, so that in the interval $c_{1} \Lambda \leq m \leq c_{2} \Lambda$ there will be the mixed phase (or equivalently, "the double Higgs phase") with two infinite size connected coherent networks of monopoles and quarks, with their averaged densities being constant over the space and following only the value of $m$.

There will be screening rather than confinement (although the difference between these two becomes to a large extent elusory here) of any test charge in this interval of $m$.

Finally, at $m=c_{2} \Lambda$ the quarks become too heavy and can not support their coherent condensate anymore, so that this last decays into independently fluctuating neutral droplets whose density and typical size decrease with increasing $m$.

At $m \gg \Lambda$ we arrive at the $N=1 S Y M$ - theory with $\Lambda_{Y M}=m^{1 / 6} \Lambda^{5 / 6}$, and with heavy $\left(m \gg \Lambda_{Y M}\right)$ quarks which are confined.

\footnotetext{
${ }^{6}$ That there are monopoles in this theory at $m \ll \Lambda$ can be seen as follows. First, let us consider the effective Lagrangian obtained by integrating out hard degrees of freedom with high energy scales $\mu \geq \mu_{o}, \mu_{o} \sim\left(\Lambda^{5} / m\right)^{1 / 4}$. These include the instanton contributions, as the typical instanton size is $\bar{\rho} \sim\left\langle Q^{\dagger} Q\right\rangle^{-1 / 2} \sim 1 / \mu_{o}$. The instanton will add the AffleckDine-Seiberg term $\Lambda^{5} /(\bar{Q} Q)$ to the original superpotential $m \bar{Q} Q$. Now, the so obtained effective Lagrangian is the appropriate one to look, in particular, for a possible string solution if the characteristic distances involved in the string formation are larger than $\bar{\rho}$.

This is the case at the classical level, and there will be the solution for the AbrikosovNielsen-Olesen like string with the magnetic flux. But because the quarks are in the fundamental representation, the gauge group is $\mathrm{SU}(2)$ which is simply connected and there are no truly uncontractable strings in this theory. This implies that the above classical string will break up on account of quantum tunneling effects. Physically, this break up will be realized through the production of a pair of magnetically charged "particle" and "antiparticle", with their subsequent separation along the string axis to screen the external infinitely heavy monopoles at the string ends. So, these magnetically charged particles should be present in the excitation spectrum of this theory (even if they are not well formed).
} 
The chiral quark condensate $\langle\bar{Q} Q\rangle \sim \Lambda^{5 / 2} / m^{1 / 2} \sim \Lambda_{Y M}^{3} / m$ is small but nonzero even in this region, but this small value is unrelated here with the gluon masses and charge screening by quarks, and is a pure quantum loop effect of heavy quarks: $\langle\bar{Q} Q\rangle \sim\langle\lambda \lambda\rangle / m$ (similarly to the heavy quark condensate $\langle\bar{\Psi} \Psi\rangle \sim\left\langle G_{\mu \nu}^{2}\right\rangle / m$ in the ordinary QCD).

\section{Broken $N=2 \quad S U(2) \mathrm{SYM}$}

Let us recall the famous solution of this theory by N. Seiberg and E. Witten, with the low energy Lagrangian (at small $\mu \ll \Lambda$ ) [12]:

$$
\begin{gathered}
L=\int d^{4} \theta\left\{M^{\dagger} e^{V_{D}} M+\bar{M}^{\dagger} e^{-V_{D}} \bar{M}+\operatorname{Im}\left(A_{D} A^{\dagger} / 4 \pi\right)\right\}-\frac{i}{16 \pi} \int d^{2} \theta \tau_{D} W_{D}^{2}+ \\
+\int d^{2} \theta\left\{\sqrt{2} \bar{M} M A_{D}+\mu U\left(A_{D}\right)\right\}+\text { h.c. }
\end{gathered}
$$

Here $M$ is the monopole field. Because it was not integrated yet, the terms entering the Lagrangian in Eq. $(5)\left(\tau_{D}\right.$, etc.) do not contain the monopole loop contributions and have no singularity at $\mu \rightarrow 0$ and $\langle U\rangle \rightarrow \Lambda^{2}$. The field $V_{D}$ is those of the dual photon and $W_{D}$ is its field strength. Below, it will be convenient for us to consider $A$ and $U$ in Eq.(5) as functions of the field $A_{D}$ which is a pure quantum field, i.e. has zero vacuum expectation value. The vacuum state we are dealing with is at $\langle U\rangle=\Lambda^{2}$, with $\tau_{D} \sim 1$ and $\operatorname{Im}\left(A_{D} A^{\dagger} / 4 \pi\right) \sim A_{D} A_{D}^{\dagger}$ in Eq. (5) at small $\mu$.

How the effective Lagrangian for these fields can look if $\mu$ is large in comparison with $\Lambda$ ? Because at $\mu \gg \Lambda$ the degrees of freedom which have been integrated out were heavy (in particular, the charged Higgs fields with their masses $\sim \mu$, and charged $W^{ \pm}$bosons with their masses $m_{W} \sim$ $\Lambda_{Y M}=\mu^{1 / 3} \Lambda^{2 / 3}$ ), the $N=2$ supersymmetry will be broken explicitly and $\mu$ dependence will penetrate the effective Lagrangian. At the same time, it is not difficult to see that due to: a) holomorphicity; b) $R$-charge conservation (with the $R$-charge of $\mu$ equal two); c) the known limit at $\mu \ll \Lambda$, the additional $\mu$ - dependence can not appear in the $F$ - terms, and so will appear in the $D$ - terms only.

Besides, restricting ourselves to the terms with no more than two spacetime derivatives, it will be sufficient for our purposes to write these D-terms for the monopole and $A_{D}$ fields as the standard kinetic terms multiplied 
by the c-number Z-factors $Z_{M}(\mu / \Lambda)$ and $Z_{H}(\mu / \Lambda)$, originating from those degrees of freedom which have been integrated out. Let us denote by $L_{\mu}$ the so obtained Lagrangian.

Recalling that the original theory was $N=2$ SYM broken by the mass term of the Higgs fields, we are ensured that at $\mu \gg \Lambda$ the Higgs fields become heavy, with their masses $m_{H} \sim \mu$, and decouple. So, we end up with $N=1$ SYM with the scale parameter: $\Lambda_{Y M}=\mu^{1 / 3} \Lambda^{2 / 3}$, and this is the only scale of this theory.

On the other hand, one obtains from $L_{\mu}$ at $\mu \gg \Lambda$ that ${ }^{7} Z_{H}$ stays intact, $Z_{H}(\mu / \Lambda \gg 1) \sim 1$, in order to have $m_{H} \sim \mu$, while the values of the dual photon and monopole masses look as

$$
m_{\tilde{\gamma}}^{2} \sim Z_{M}(\mu / \Lambda)\left\langle 0\left|M^{\dagger} M\right| 0\right\rangle \sim Z_{M}(\mu / \Lambda) \cdot \mu \Lambda, \quad m_{M}^{2} \sim Z_{M}^{-2}(\mu / \Lambda) \cdot \Lambda^{2} .
$$

Let us combine now Eq.(6) with the additional assumption: there is no massless particles in the spectrum of $N=1$ SYM. Then, this requires ${ }^{8}$ :

$$
Z_{M}(\mu / \Lambda) \sim\left(\frac{\Lambda}{\mu}\right)^{1 / 3} \text { at } \mu \gg \Lambda
$$

It follows now from Eqs.(6), (7):

$$
m_{\tilde{\gamma}} \sim m_{M} \sim \Lambda_{Y M} \quad \text { at } \quad \mu \gg \Lambda
$$

i.e. both the dual photon and monopole survive in the spectrum of the $N=1$ SYM. This is nontrivial in the sense that one of them or both could become heavy and decouple at $\mu \gg \Lambda$.

As for the value of the monopole condensate, it depends clearly on the normalization of the monopole field. In the presence of the monopole $Z_{M}$ factor, the old normalization $\langle 0|\bar{M} M| 0\rangle \sim \mu \Lambda$ is not the natural one. The appropriate normalization is: $\langle 0|\bar{N} N| 0\rangle=\left\langle 0\left|Z_{M}^{1 / 2} \bar{M} \cdot Z_{M}^{1 / 2} M\right| 0\right\rangle$, and it has

\footnotetext{
${ }^{7}$ In the above described set up, there is no need for the function $U\left(A_{D}\right)$ of the $\mu U\left(A_{D}\right)$ term in Eq.(5) to be exactly the Seiberg-Witten function. For our purposes and for simplicity, it will be sufficient to keep only three first terms (i.e. constant, linear and quadratic in $\left.A_{D}\right)$ in the expansion of $U\left(A_{D}\right)$ in powers of $A_{D}$, to ensure that the adjoint Higgs becomes heavy, $m_{H} \sim \mu$, and decouples.

${ }^{8}$ If $(\mu / \Lambda)^{1 / 3} \cdot Z_{M}(\mu / \Lambda) \rightarrow 0$, the dual photon will be massless (on the scale $\Lambda_{Y M}$, i.e. $\left.m_{\tilde{\gamma}} / \Lambda_{Y M} \rightarrow 0\right)$, while if $(\mu / \Lambda)^{1 / 3} \cdot Z_{M}(\mu / \Lambda) \rightarrow \infty$ the monopole will be massless.
} 
the right scale: $\langle 0|\bar{N} N| 0\rangle \sim \Lambda_{Y M}^{2} \cdot{ }^{9}$

On the whole, the above described results were obtained implying that there is no phase transition in the broken $N=2$ SYM theory when going from small $\mu \ll \Lambda$ to large $\mu \gg \Lambda$ (in a sense, at least, that monopoles continue to form the coherent condensate which gives the mass to the dual photon; on the other hand, restructuring of the spectrum definitely occurs at $\mu \sim \Lambda$ ). They show a selfconsistency of this assumption and give a strong support to the widely accepted expectation that the $N=1 \mathrm{SYM}$ theory is confining, with the confining mechanism those of the dual superconductor. In other words, when going from $\mu \ll \Lambda$ to $\mu \gg \Lambda$ in the broken $N=2 S Y M$ theory, the "external" adoint Higgs of this theory decouples at $\mu \sim \Lambda$ and its role is taken by the dynamically formed and condensing "internal" composite adjoint Higgs of the $N=1 S Y M$ - theory, while monopoles continue to form the coherent condensate and keep the dual photon massive.

\section{Broken $N=2 S U(2), N_{F}=1 S Q C D$}

The solution of the unbroken $N=2$ theory has been given by N. Seiberg and E. Witten [13]. The original superpotential of the broken $N=2 \rightarrow N=$ 1 theory has the form (the kinetic terms are canonical):

$$
W=m \bar{Q} Q+h \sqrt{2} \bar{Q} \frac{\tau^{a}}{2} \phi^{a} Q+\mu \phi^{2},
$$

where the quark fields $Q$ and $\bar{Q}$ are in the $\mathbf{2}$ and $\overline{\mathbf{2}}$ representations of the colour group $S U(2)$, and $\phi$ is the adjoint Higgs field. The unbroken $N=2$ SUSY corresponds to $\mu=0$ and $h=1$.

The properties of this broken $N=2$ theory have been considered previously in $[14-16]$. The most detailed description has been given recently by A. Gorsky, A. Vainshtein and A. Yung in [17], and we use widely the results

\footnotetext{
${ }^{9}$ It is not difficult to trace the role of higher order terms $\sim A_{D}^{2}\left(A_{D} / \Lambda\right)^{k \geq 1}$ in the expansion of $U\left(A_{D}\right)$ in $L_{\mu}$ in powers of $\left(A_{D} / \Lambda\right)$. After integrating out the heavy field $A_{D}$, these will give additional terms in the superpotential in powers of the pure quantum field $(\bar{M} M-\mu \Lambda) / \mu \Lambda$. Rescaling the monopole field to $N=Z_{M}^{1 / 2} M$, to have the canonical kinetic term for the field $N$, these additional terms in the superpotential will come in powers of the pure quantum field $\left(\bar{N} N-\Lambda_{Y M}^{2}\right) / \Lambda_{Y M}^{2}$, i.e. depending only on the scale $\Lambda_{Y M}$, as it should be in the $N=1 \mathrm{SYM}$ theory.
} 
of this paper below. For our purposes, we will deal with the special case of light quarks weakly coupled to the Higgs field:

$$
m \ll \Lambda, \quad \sigma=h\left(\frac{\Lambda}{m}\right)^{3 / 2} \ll 1,
$$

where $\Lambda$ is the scale parameter of the original fundamental theory $(\Lambda=1$ in what follows). Under the conditions of Eq.(10), one vacuum state decouples and there remain two physically equivalent vacuum states. So, it will be sufficient to deal with one of them where the condensates of original fields take the values [17]:

$$
\langle\bar{Q} Q\rangle \sim \mu m^{-1 / 2} ; \quad\langle U\rangle=\left\langle\phi^{2}\right\rangle \sim m^{1 / 2} ; \quad\langle\lambda \lambda\rangle \sim \mu m^{1 / 2},
$$

while the condensate of the monopole field is

$$
\langle\bar{M} M\rangle \sim \mu m^{1 / 4} .
$$

Under the conditions of Eq.(10), the only freedom remained is the relative value of $\mu$ and $m$, and the phase and physical content of this theory depend essentially on this. Indeed:

a) at sufficiently small $\mu$; the quarks $Q(\bar{Q})$ are "heavy" and decouple, we are in the pure $N=2 \mathrm{SYM}$ - theory with $\Lambda_{\text {eff }}^{(1)}=m^{1 / 4} \Lambda^{3 / 4}$, broken by the small $\mu U$ - term. The vacuum is the Seiberg-Witten vacuum, i.e. the dominant condensate is the Higgs one, $\left\langle\phi^{a}\right\rangle$, leading to $S U(2) \rightarrow U(1)$, with $W^{ \pm}$masses $M_{W} \sim \Lambda_{\text {eff }}^{(1)} \sim m^{1 / 4}$. The light monopole field condenses in the low energy $U(1)$ - theory, resulting in the confinement of electric charges. The lightest particles are the dual photon $\tilde{\gamma}$, its $N=2$ partner $A_{D}$ and the monopole composite $(\bar{M} M)^{1 / 2}$, all with small masses $\sim \mu^{1 / 2} m^{1 / 8}$. We will call this phase the magnetic one.

b) at sufficiently large $\mu$; the Higgs field $\phi$ is heavy and decouples, we are in $N=1, N_{F}=1 \mathrm{SQCD}$ with $\Lambda_{e f f}^{(2)}=\mu^{2 / 5} \Lambda^{3 / 5}$ and with light quark composite $(\bar{Q} Q)^{1 / 2}$ - fields, with their masses $\sim m \ll \Lambda_{\text {eff }}^{(2)}$. Here, the large quark condensate $\left\langle Q_{i}\right\rangle \sim\left\langle\bar{Q}^{j}\right\rangle$ dominates, $\mathrm{SU}(2)$ is broken completely and there is confinement of magnetically charged excitations (monopoles), see the footnote 6 . The low energy effective Lagrangian is those of Affleck-Dine-Seiberg [11]. We will call this phase the electric one.

So, unlike the examples considered in previous sections, at the conditions given by Eq.(10) we have a good control here over the phases of our theory 
in both limiting cases of small and large values of $\mu$, and these phases are dual to each other and are dominated by coherent condensates of mutually non-local monopole and quark fields. Our purpose now is to trace in more detail the transition between the magnetic and electric phases at some value $\mu \simeq \mu_{o}$, when going from small $\mu \ll \mu_{o}$ to large $\mu \gg \mu_{o}$ values of $\mu$. We expect that this transition proceeds through the formation of the mixed phase in some region $c_{1} \mu_{o} \leq \mu \leq c_{2} \mu_{o}$ (with $c_{1} \leq c_{2}$, but parametrically both $\left.c_{1} \sim c_{2} \sim O(1)\right) .10$

In the magnetic phase region $0 \leq \mu \lesssim \mu_{o}$ we will proceed in the same way as in the previous section, by retaining only the lightest fields of the dual photon $\tilde{\gamma}, A_{D}$ and monopole $M$. All quark fields, in particular, are integrated out. Although, see Eq.(10), $h \rightarrow 0$ and quarks do not interact directly with the Higgs fields, they interact with massive charged gluons and gluinos and will give corrections in powers of the characteristic scale $\sim\langle\lambda \lambda\rangle / m^{3} \sim$ $\left(\mu / m^{5 / 2}\right)$. Further, being integrated out, the massive gluons and gluinos will transmit these corrections to the monopole $Z_{M}$-factor: $Z_{M}=Z_{M}\left(\mu / m^{5 / 2}\right)$. So, the quarks really decouple only at $\mu \ll m^{5 / 2}$ where $Z_{M}\left(\mu / m^{5 / 2} \rightarrow 0\right) \rightarrow 1$, while at $\mu>m^{5 / 2}$ the quarks influence the physics and $Z_{M} \neq 1$.

Similarly, in the electric phase region $\mu_{o} \lesssim \mu$, after integrating out the Higgs and gauge fields, the quark $Z_{Q}$-factor will obtain corrections in powers of the characteristic scale $\sim\langle\lambda \lambda\rangle / \mu^{3} \sim\left(m^{1 / 2} / \mu^{2}\right)$, so that $Z_{Q}=Z_{Q}\left(m^{1 / 4} / \mu\right)$ and the heavy Higgs field really decouples only at $\mu \gg m^{1 / 4}: Z_{Q}\left(m^{1 / 4} / \mu \rightarrow\right.$ $0) \rightarrow 1$. At $\mu_{o} \lesssim \mu<m^{1 / 4}$ the adjoint Higgs field still influences the physics, and $Z_{Q} \neq 1$.

It is not difficult to see that with the choice:

$$
Z_{M}^{o} \equiv Z_{M}\left(\mu_{o} / m^{5 / 2}\right) \sim \frac{m^{1 / 4}}{\mu_{o}}, \quad Z_{Q}^{o} \equiv Z_{Q}\left(m^{1 / 4} / \mu_{o}\right) \sim \frac{m}{\mu_{o}},
$$

all particle masses and all (properly normalized) condensates are matched in the transition region $\mu \simeq \mu_{o}$, as it should be (the gauge couplings are $\sim 1$ at

\footnotetext{
${ }^{10}$ Here and in other supersymmetric theories, the condensates of chiral superfields are frequently simplest smooth functions of chiral parameters in the whole parameter space, see for instance Eqs.(11), (12). This does not contradict to possibility for a system to be in qualitatively different (dual to each other) phases at different values of parameters, because these condensates are not order parameters for these phases. Rather, the masses of the direct and dual photon (together with $W^{ \pm}$- masses) look more like the order parameters in the electric and magnetic phases respectively.
} 
$\mu \sim \mu_{o}$, see below):

$$
\begin{gathered}
M_{H} \sim M_{M} \sim M_{Q} \sim \mu_{o} ; \quad M_{W^{ \pm}} \sim M_{\gamma} \sim M_{\tilde{\gamma}} \sim m^{1 / 4}>\mu_{o} \\
\left\langle\phi^{2}\right\rangle \sim\left\langle\sqrt{Z_{Q}^{o}} \bar{Q} \cdot \sqrt{Z_{Q}^{o}} Q\right\rangle \sim\left\langle\sqrt{Z_{M}^{o}} \bar{M} \cdot \sqrt{Z_{M}^{o}} M\right\rangle \sim m^{1 / 2} .
\end{gathered}
$$

The nontrivial fact is that the number of matching conditions in Eqs. (14) (15) is larger than the choice of only two numbers in Eq.(13).

As for the value of $\mu_{o}$, it is determined by matching at $\mu \sim \mu_{o}$ of two characteristic scales: those of $\Lambda_{e f f}^{(1)}=m^{1 / 4}$ from the magnetic phase region $0<\mu<\mu_{o}$, and those of $\Lambda_{\text {eff }}^{(2)}=\mu^{2 / 5}$ from the electric phase region $\mu>\mu_{o}$ :

$$
m^{1 / 4} \sim \mu_{o}^{2 / 5} \rightarrow \mu_{o} \sim m^{5 / 8}
$$

It is not difficult to see that this is equivalent to matching of the gauge couplings. Indeed, in the electric phase region the charged degrees of freedom decouple at the scale $\sim M_{W}=M_{\gamma}$, determined by the quark condensation, restructuring the spectrum and decoupling of the light neutral quark composite field $(\bar{Q} Q)^{1 / 2}$. So, the inverse gauge coupling behaves as $\tau \sim \ln \left(M_{W} / \Lambda_{\text {eff }}^{(2)}\right)$, which is $\tau^{o} \sim \ln \left(m^{1 / 4} / \mu_{o}^{2 / 5}\right)$ at $\mu \sim \mu_{o}$ (see Eq. (14)).

In the magnetic phase region there are two characteristic scales: a) those connected with the Higgs condensation and decoupling of $W^{ \pm}$and gives $\tau \sim$ $\ln \left(M_{W} / \Lambda_{\text {eff }}^{(1)}\right) \sim 1$; and b) those connected with the monopole condensation and decoupling of the neutral monopole composite field $(\bar{M} M)^{1 / 2}$. This last gives $\tau_{D} \sim \ln \left(\Lambda_{\text {eff }}^{(1)} / M_{\tilde{\gamma}}\right)$, which is also $\tau_{D}^{o} \sim 1$ at $\mu \sim \mu_{o}$ (see Eq.(14)). Therefore, the matching of couplings at $\mu \sim \mu_{o}$ gives: $\tau^{o} \sim \tau_{D}^{o} \sim 1 \sim$ $\ln \left(m^{1 / 4} / \mu_{o}^{2 / 5}\right)$.

So, $Z_{M}$ behaves like $Z_{M}=\left[1+\left(\mu / m^{5 / 2}\right)\right]^{1 / 5}$ in the magnetic phase region $0<\mu \lesssim m^{5 / 8}$, and the monopole mass (or, more exactly, the mass of the monopole composite field $\left.(\bar{M} M)^{1 / 2}\right)$ is: $M_{M} \sim \mu^{2 / 5} m^{3 / 8}$ at $m^{5 / 2}<\mu<m^{5 / 8}$, while $Z_{Q}$ behaves like $Z_{Q}=\left[1+\left(m^{1 / 4} / \mu\right)\right]^{-1}$ in the electric phase region $m^{5 / 8} \lesssim \mu$.

It is seen also from Eqs.(13) and (16) that there is a kind of duality relation at the transition point $\mu \sim \mu_{o}: Z_{M}^{o} \sim 1 / Z_{Q}^{o}$. Moreover, at small $\mu \ll m^{5 / 2}$ the confinement is very weak and the quark is nearly free, with mass $M_{Q}=m$ and $Z_{Q} \rightarrow 1$. So, $Z_{M} \sim 1 / Z_{Q}$ holds also at small $\mu$ as well. Therefore, because the behaviour within the same phase region is smooth, 
the duality relation $Z_{Q} \sim 1 / Z_{M}$ holds in the whole magnetic phase region $0<\mu<\mu_{o}$.

Introducing the effective quark mass as: $M_{Q}^{(e f f)}=Z_{Q}^{-1} \cdot m$, it is then: $M_{Q}^{(e f f)} \sim Z_{M} \cdot m$, and $\left(M_{M} / M_{Q}^{(e f f)}\right) \sim\left(\mu / \mu_{o}\right)^{1 / 5}<1$ at $m^{5 / 2}<\mu<\mu_{o}$. This shows that quark is heavier than monopole in the whole magnetic phase region, and this is self consistent.

As was pointed out above, the example considered in this section has an advantage that we have a good control over the properties of the magnetic and electric phases at both sides, $\mu \ll m^{5 / 8}$ and $\mu \gg m^{5 / 8}$, of the transition region at $\mu \sim m^{5 / 8}$. As for the properties of the mixed phase in the transition region, they are similar to those described in previous sections. In short:

1) At very small $\mu<m^{5 / 2}$ the condensates of the Higgs and monopole fields dominate, with the coherent Higgs condensate responsible for the $W^{ \pm}$ masses $M_{W}$, and the coherent monopole condensate responsible for the dual photon mass $M_{\tilde{\gamma}}$. The quarks are "heavy" and confined, and there are rare incoherent fluctuations of neutral quark-antiquark pairs.

2) The density of these neutral quark pairs increases with increasing $\mu$.

3) These quark bags (or strings) percolate at $\mu=c_{1} m^{5 / 8}$. At $c_{1} m^{5 / 8} \leq$ $\mu \leq c_{2} m^{5 / 8}$, with $c_{1,2} \sim O(1)$, the system is in the mixed phase, where two infinite size connected coherent networks of electric and magnetic strings (or bags) coexist. In this region (parametrically): quark condensate $\sim$ Higgs condensate $\sim$ monopole condensate, quark mass $\sim$ Higgs mass $\sim$ monopole mass, photon mass $\sim$ dual photon mass, etc. (see Eqs. (14)-(15)).

4) At $\mu=c_{2} m^{5 / 8}$ the quark condensate takes over and enforces depercolation of the connected coherent condensates of the monopole and Higgs fields. I.e., these last decay into separate independently fluctuating droplets, whose density and typical size decrease with increasing $\mu$, while the gluon masses originate now from the coherent quark condensate.

5) At $\mu>m^{1 / 4}$ the Higgs field becomes too heavy and decouples completely, while the magnetically charged excitations are heavy and confined into rare small size neutral pairs.

\section{Summary}

As has been argued on a number of examples above, the mixed phases exist with their properties qualitatively different from those of pure phases. 
And the appearance of mixed phases is not an exception but rather a typical phenomenon in various gauge theories, both supersymmetric and ordinary.

\section{Conclusion}

Dear Arkady, be healthy and happy!

\section{Acknowledgements}

I am grateful to the Organizing Committee of "QCD-2002/Arkadyfest" for a kind hospitality and support. 


\section{References}

[1] E. Witten, Nucl. Phys. B149, 285 (1979); Ann. Phys. (N.Y.) 128, 363 (1980)

[2] E. Witten, Phys. Rev. Lett. 81, 2862 (1998); [hep-th/9807109]

[3] V. Chernyak, Preprint BINP 98-61 (1998) (revised) [hep-th/9808092]

[4] E. Witten, Phys. Lett. B86, 283 (1979)

[5] S. Coleman, Ann. Phys. (N.Y.) 101 (1976) 239

[6] G. Veneziano and S. Yankielowicz, Phys. Lett. B113, 321 (1982)

[7] M.A. Shifman and A.I. Vainshtein, Nucl. Phys. B296, 445 (1988)

[8] V. Chernyak, Phys. Lett. B450, 65 (1999) [hep-th/9808093]

[9] E. Witten, Nucl. Phys. B507, 658 (1997); [hep-th/9706109]

[10] I.I. Kogan, A. Kovner and M. Shifman, Phys. Rev. D57, 5195 (1998); [hep-th/9712046]

[11] I. Affleck, M. Dine and N. Seiberg, Phys. Lett. B137, 187 (1984)

[12] N. Seiberg and E. Witten, Nucl. Phys. B426, 19 (1994) [hepth/9407087]

[13] N. Seiberg and E. Witten, Nucl. Phys. B431, 484 (1994) [hepth/9408099]

[14] K. Intrilligator and N. Seiberg, Nucl. Phys. B431, 551 (1994) [hepth/9408155]

[15] S. Elitzur, A. Forge, A. Giveon and E. Rabinovici, Phys. Lett. B353, 79 (1995) [hep-th/9504080]; Nucl. Phys. B459, 160 (1996) [hepth/9509130]

[16] S. Elitzur, A. Forge, A. Giveon, K. Intrilligator and E. Rabinovici, Phys. Lett. B379, 121 (1996) [hep-th/9603051]

[17] A. Gorsky, A. Vainshtein and A. Yung, Nucl. Phys. B584, 197 (2000) [hep-th/0004087] 